\title{
Deeply Virtual Compton Scattering at HERMES
}

\author{
Hrachya Marukyan \\ for the HERMES Collaboration \\ CGSWHP06, Tbilisi, Georgia, September 4-8, 2006
}

- Motivation: Spin Composition of the Nucleon

- Generalized Parton Distributions and DVCS process

- DVCS Measurement at HERMES

- HERMES Results on Azimuthal Asymmetries

- Summary and Outlook 


\section{Nucleon Spin Composition}

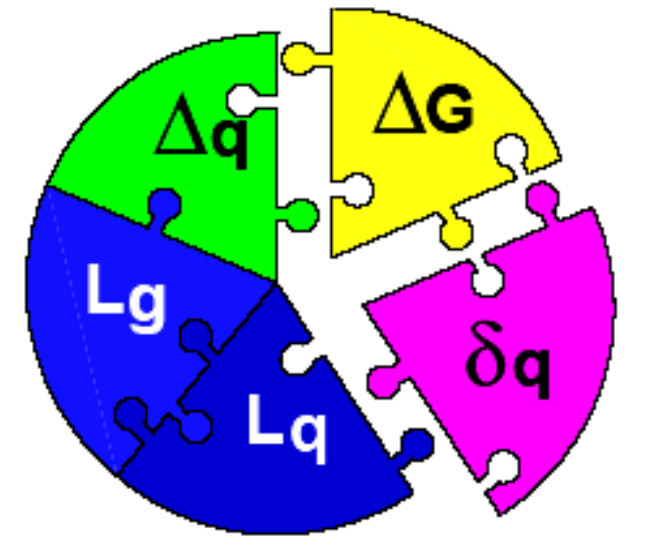

$$
\begin{gathered}
\frac{1}{2}=\underbrace{\frac{1}{2} \overbrace{(\Delta u+\Delta d+\Delta s)}^{\Delta \Sigma}+L_{q}}_{J_{q}}+\underbrace{\Delta G+L_{g}}_{J_{g}} \\
\Delta \Sigma \sim 20-35 \%: \begin{array}{c}
\text { MEASURED IN DIS } \\
\text { HERMES } \sim 0.3
\end{array} \\
\Delta G: \text { FIRST MEASUREMENTS } \\
L_{q}, L_{g}: \text { UNKNOWN! }
\end{gathered}
$$

Ji's Relation: Total angular momentum — Ji, PRL 78 (1997) 610

$$
J_{q, g}=\lim _{t \rightarrow 0} \frac{1}{2} \int_{-1}^{1} d x x \underbrace{\left[H_{q, g}+E_{q, g}\right]}_{G P D s}
$$

$\Longrightarrow$ The HUNT FOR $L_{q}$ 


\section{GPDs and DVCS Process}

GPDS ACCESSIBLE IN HARD EXCLUSIVE PROCESSES:

DVCS: HARD PHOTOPRODUCTION OF A REAL PHOTON $\left(\gamma^{*} N \rightarrow N^{\prime} \gamma\right)$, VIRTUAL PHOTON GENERATED BY LEPTON SCATTERING $\Rightarrow e N \rightarrow e^{\prime} N^{\prime} \gamma$

FACTORIZATION THEOREM:

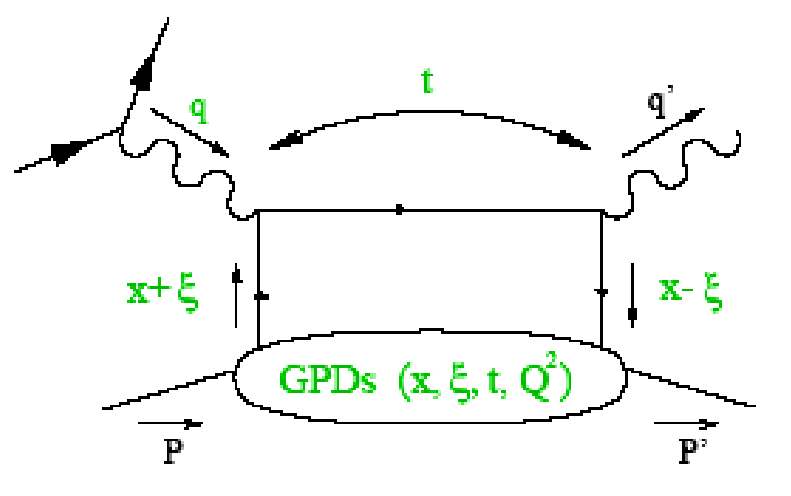

$x \pm \xi:$ PARTON LONGITUDINAL MOMENTUM FRACTIONS

$\xi:$ FRACTION OF THE MOMENTUM TRANSFER, $\xi \simeq \frac{x_{B}}{2-x_{B}}$,

$t$ : INVARIANT MOMENTUM TRANSFER, $t \equiv\left(p-p^{\prime}\right)^{2}$

NUCLEON STRUCTURE:

$H_{q}, \widetilde{H}_{q}-$ CONSERVE NUCLEON HELICITY

$$
\begin{gathered}
G P D s: \quad H_{q}, \widetilde{H}_{q}, E_{q}, \widetilde{E}_{q} \\
G P D s \rightarrow P D F s \\
H_{q}(x, 0,0)=q(x) \\
\widetilde{H}_{q}(x, 0,0)=\Delta q(x) \\
G P D s \rightarrow \quad F F s \\
\int_{-1}^{1} d x H_{q}(x, \xi, t)=F_{1}^{q}(t), \\
\int_{-1}^{1} d x E_{q}(x, \xi, t)=F_{2}^{q}(t)
\end{gathered}
$$$$
E_{q}, \widetilde{E}_{q}-\text { FLIP NUCLEON HELICITY, }
$$$$
\text { NOT ACCESSIBLE IN DIS }
$$ 
DVCS ( $a$ ) AND Bethe-Heitler (BH) (b) PROCESSES EXPERIMENTALLY INDisTinguishaBLE
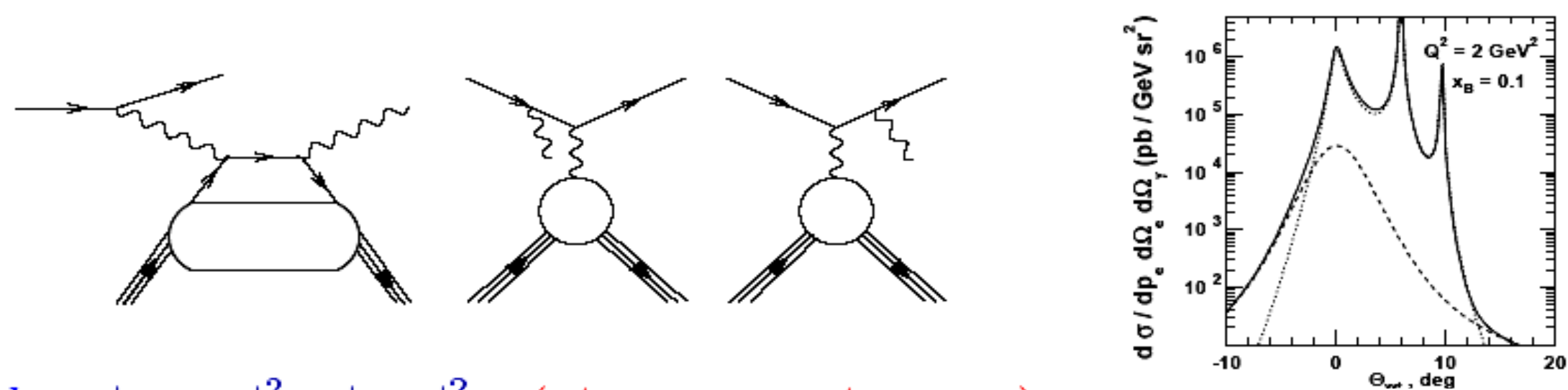

$d \sigma \propto\left|\tau_{\mathrm{DVCS}}\right|^{2}+\left|\tau_{\mathrm{BH}}\right|^{2}+\underbrace{\left(\tau_{\mathrm{DVCS}}^{*} \tau_{\mathrm{BH}}+\tau_{\mathrm{BH}}^{*} \tau_{\mathrm{DVCS}}\right)}_{I}$

HERMES KINEMATICS:

$\left|\tau_{\mathrm{DVCS}}\right|^{2}<<\left|\tau_{\mathrm{BH}}\right|^{2}$

DVCS AMPlitudes: ACCESSIBLE THROUGH AZIMUTHAL ASYMMETRIES Via I (GPDs ENTER IN LINEAR COMBINATIONS IN AMPLITUDES)

-Beam-Spin Asymmetry (BSA):

$$
d \sigma\left(\overrightarrow{e^{+}} p\right)-d \sigma\left(\overleftarrow{e^{+}} p\right) \propto \operatorname{Im}\left[F_{1} \mathcal{H}\right] \times \sin (\phi)
$$

-Beam-Charge Asymmetry (BCA):

$$
d \sigma\left(e^{+} p\right)-d \sigma\left(e^{-} p\right) \propto \operatorname{Re}\left[F_{1} \mathcal{H}\right] \times \cos (\phi)
$$

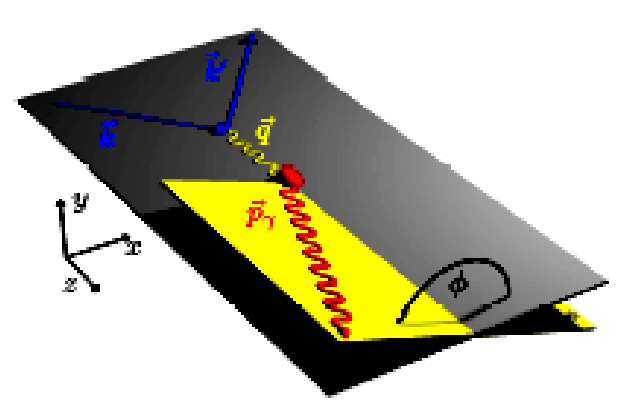




\section{The HERMES Experiment}

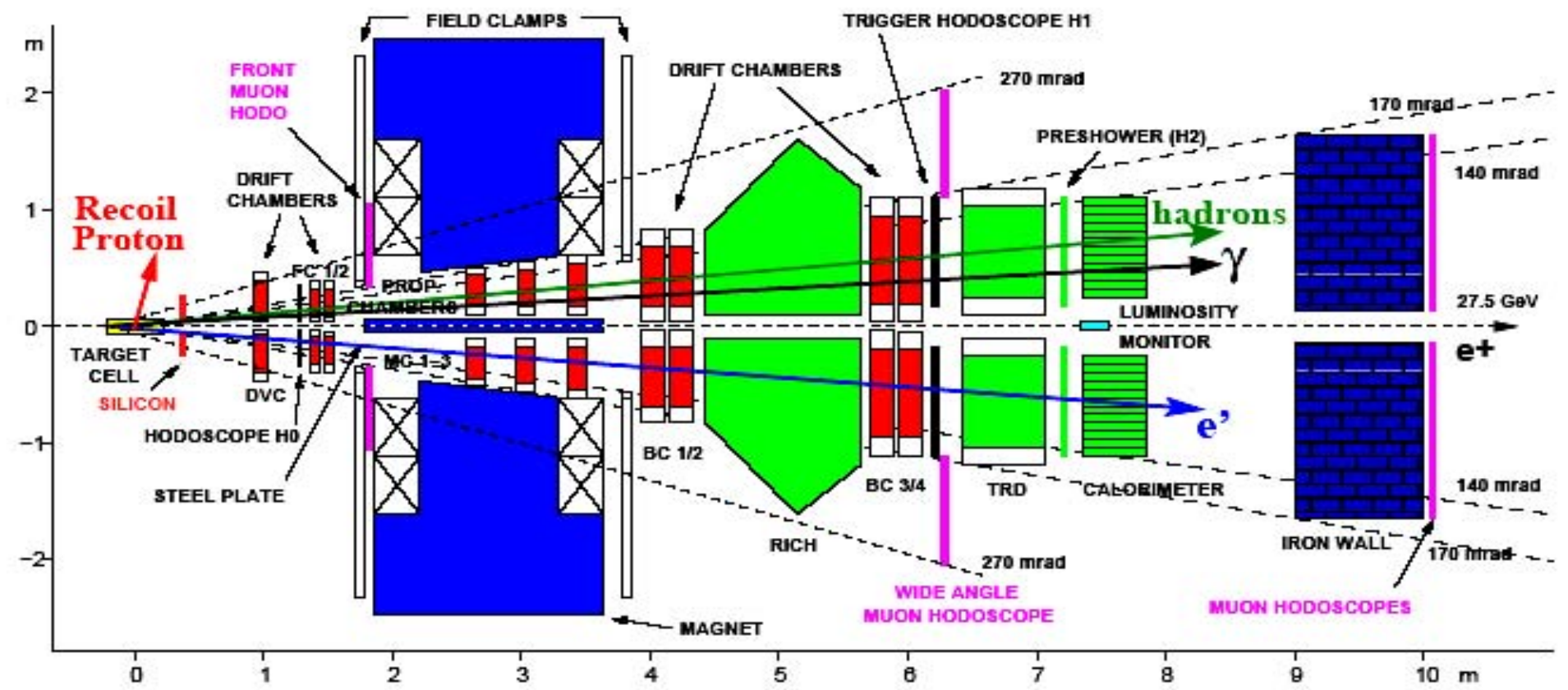

Gas TARGET:

-LONG. POLARIZED $H, D$

-UNPOLARIZED $H, D, N e, K r, X e$

- TRANSVERSELY POLARIZED $H$

$$
\left\langle\left|P_{T}\right|\right\rangle \approx 85 \%
$$

BEAM:

-LONG. POLARIZED $e^{+}$AND $e^{-}$ -ENERGy 27.6 GEV

-BOTH HELICITIES

$$
\left\langle\left|P_{B}\right|\right\rangle \approx 55 \% \text { (HERA I) }
$$

PID: $\epsilon_{e}>99 \%, \delta P / P<2 \%, \delta \theta<1 \mathrm{mrad}, \delta E_{\gamma} / E_{\gamma} \approx 5 \%$. 
-EVENTS WITH EXACTLY ONE DIS - LEPTON AND EXACTLY ONE TRACKLESS CLUSTER IN THE CALORIMETER.

-No RECOIL DETECTION $\Rightarrow$ EXCLUSIVITY VIA MISSING MASS: $M_{X}^{2}=\left(q+P-q^{\prime}\right)^{2}$

MC FOR BACKGROUND AND CUTS

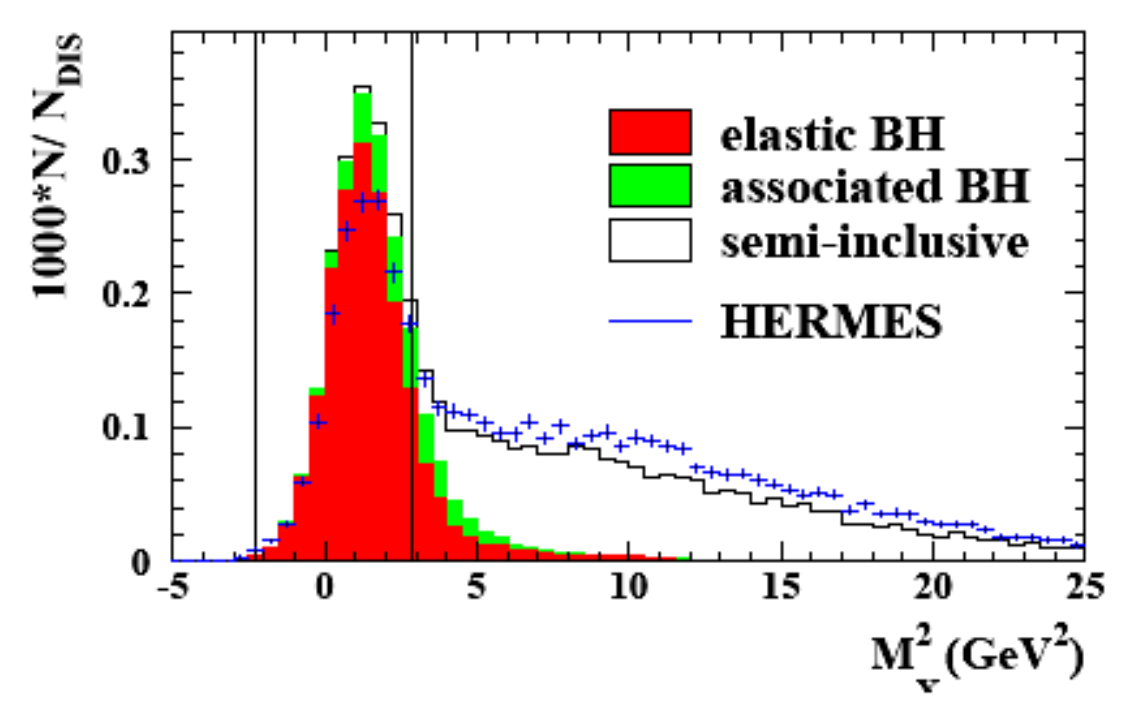

$$
e p \rightarrow e^{\prime} \gamma X
$$

Contributed Processes:

$e p \rightarrow e^{\prime} p \gamma ;$ ELASTIC BH

$e p \longrightarrow e^{\prime} \Delta^{+} \gamma ;$ Associated BH

$e p \rightarrow e^{\prime} \pi^{0} X \quad ;$ Semi-INCLUSIVE

CORRECTION ON BACKGROUND $\Rightarrow$ Mostly Decay Photons From SEMI-INCLUSIVE $\pi^{0}(\approx 6 \%)$

BACKGROUND CONTRIBUTION OVERALL $\approx 15 \%$

$\Rightarrow$ ExClusive BIN $\left(-(1.5)^{2}<M_{X}^{2}<(1.7)^{2} \mathrm{GeV}^{2}\right)$ 


$$
A_{\mathrm{LU}}(\phi)=\frac{1}{\left\langle\left|P_{b}\right|\right\rangle} \frac{\vec{N}(\phi)-\overleftarrow{N}(\phi)}{\vec{N}(\phi)+\overleftarrow{N}(\phi)} \propto \frac{\operatorname{Im} \mathcal{H}_{1}}{F_{1}} \sin \phi
$$

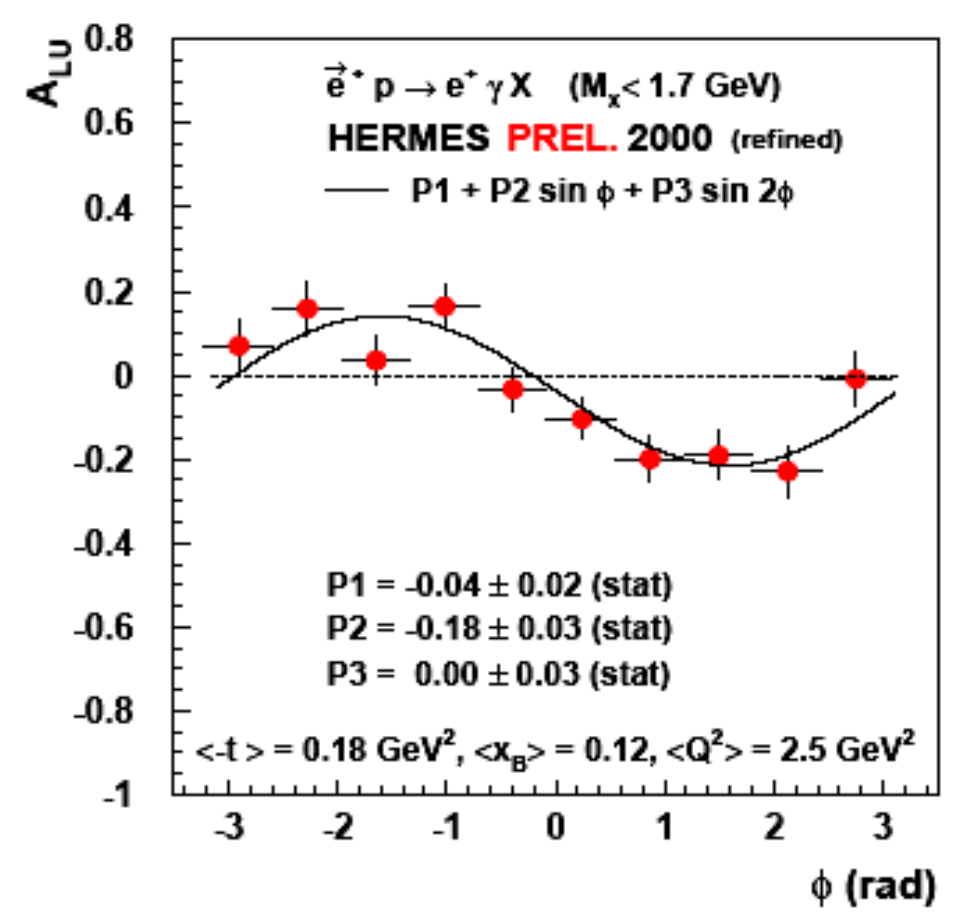

$A_{\text {LU }}$ IN EXClusive BIN: Expected $\sin (\phi)$ DEPENDENCE $\Longrightarrow \operatorname{Im} \mathcal{H}$

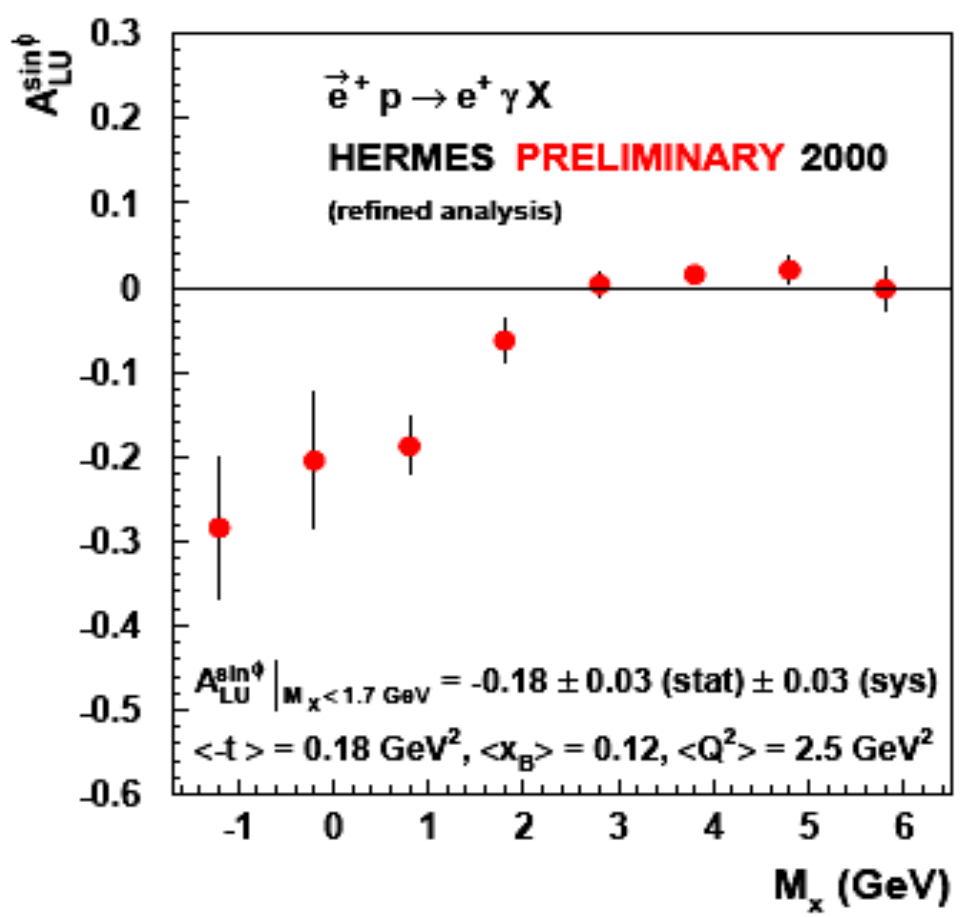

$\sin (\phi)$-MOMENTS IN NON-EXCLUSIVE REGION IS SMALL AND POSITIVE

BSA RESULTS: HERMES-PRL 87, 182001 (2001) 


\section{Beam-Charge Asymmetry on Proton}

$$
A_{\mathrm{C}}(\phi)=\frac{N^{+}(\phi)-N^{-}(\phi)}{N^{+}(\phi)+N^{-}(\phi)} \propto \frac{\operatorname{Re} \mathcal{H}}{F_{1}} \cos \phi
$$

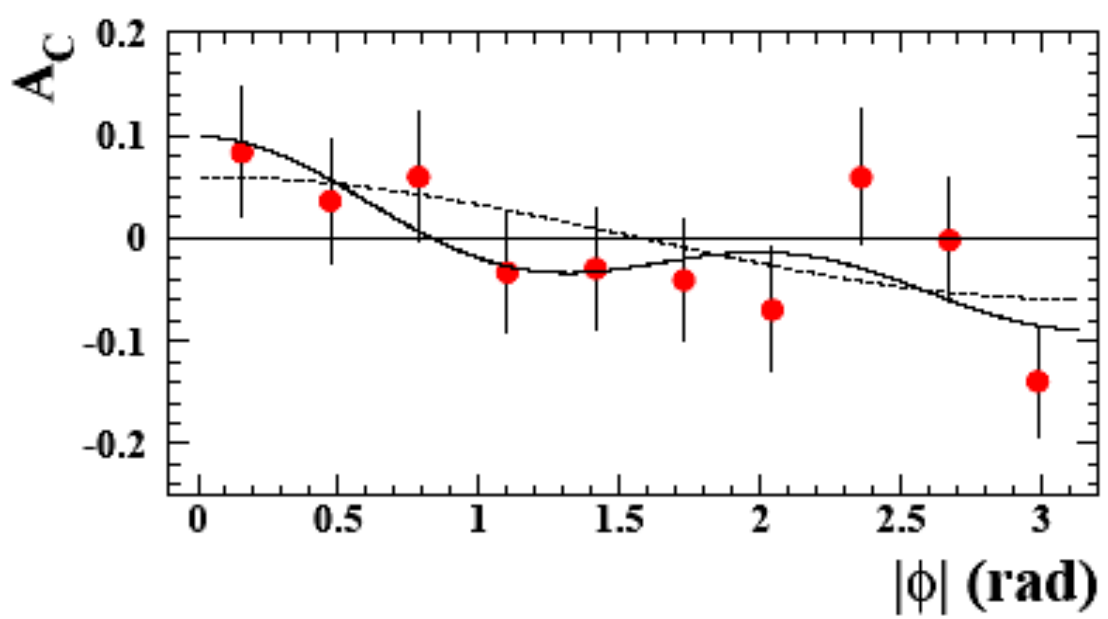

'SYMMETRIZED' BCA IN EXCLUSIVE BIN $(\phi \rightarrow|\phi|) \Longrightarrow$ CANCEL SINUSOIDAL TERMS (DUE TO Polarized BEAM)

THE SOLID CURVE $\rightarrow$ 4-PARAMETER FIT:

$P_{0}+P_{1} \cos \phi+P_{2} \cos 2 \phi+P_{3} \cos 3 \phi$

THE DASHED CURVE $\rightarrow$ PURE $\cos \phi$

EXPECTED $\cos \phi$ DEPENDENCE

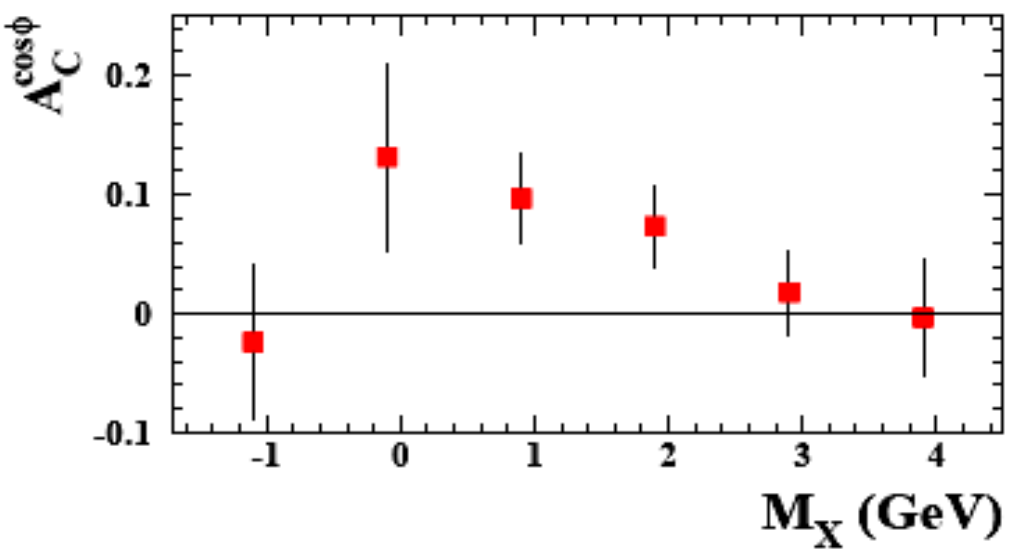

$\cos (\phi)$-MOMENTS ZERO AT HIGHER MISSING MASS

$$
\begin{gathered}
A_{\mathrm{C}, \text { Proton }}^{\cos (\phi)}= \\
0.063 \pm 0.029 \text { (STAT. }) \pm 0.026 \text { (sYs. }) \\
\hline
\end{gathered}
$$

A. Airapetian et al., HeP-EX/0605108, SUBMitTed TO PRL 


\section{Comparison to model calculations}

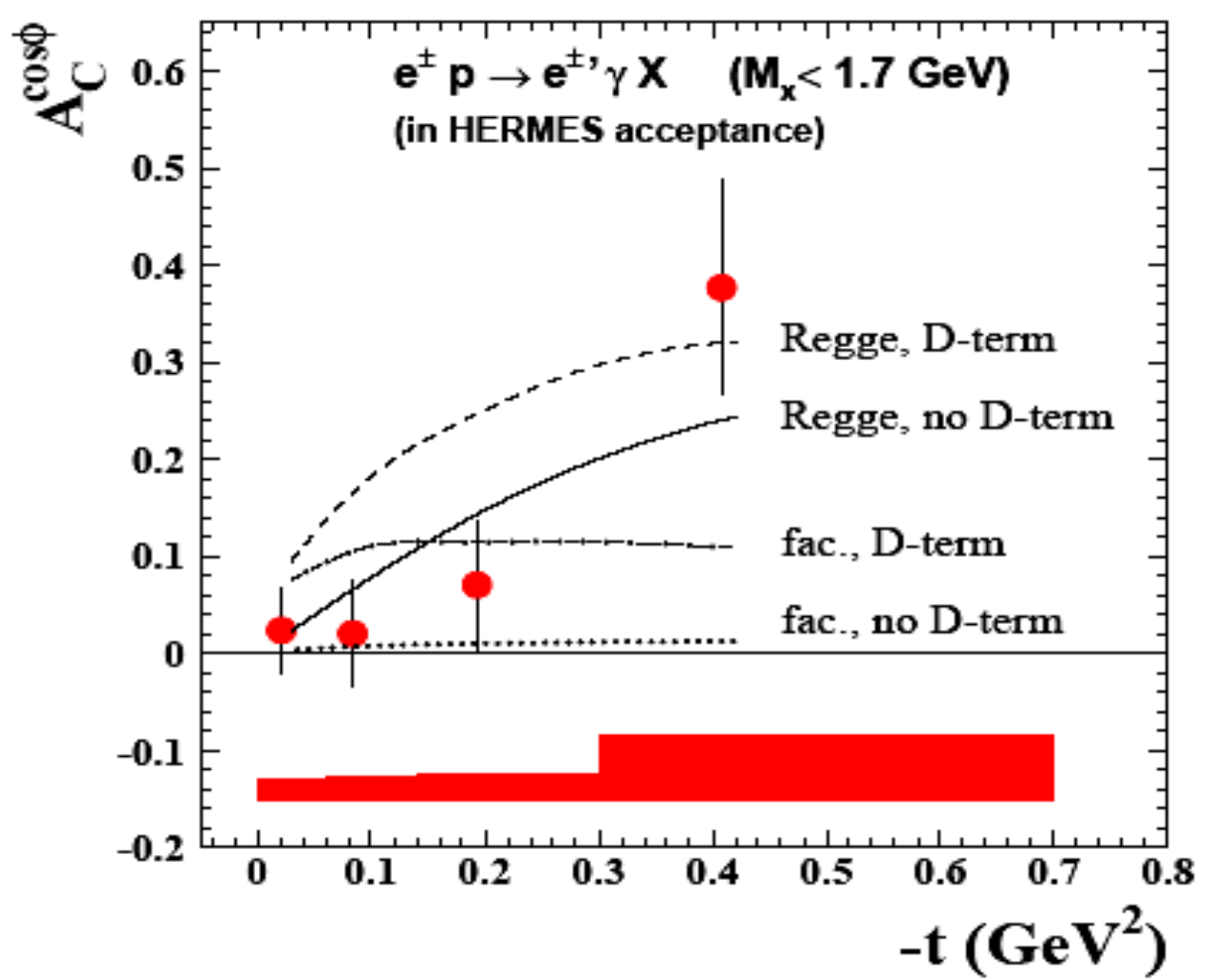

GPD MODELS: M. VANDERHAEghen et al.,

Phys. Rev. D60 (1999) 094017; K. Goeke et al.

Prog. Part. Nucl. Phys. 47 (2001) 401.

- GPD H DOMINATES, E SUPPRESSED

- Curves: 4 Different parameter Sets

- Model Calculations at aVERage KINEMATIC VALUES PER BIN

IN LAST $t$-BIN: LARGE CONTRIBUTION FROM THE ASSOCIATED PRODUCTION (NOT INCLUDED IN MODEL CALCULATION) $\Rightarrow$ DATA DISFAVOR REGGE-INSPIRED $t$-DEPENDENCE WITH D-TERM SMALL $e^{-} p$ SAMPLE $\left(L \approx 10 \mathrm{~PB}^{-1}\right)$ $\Rightarrow t$-DEPENDENCE OF BCA $\rightarrow$ POSSIBLE TOOL TO CONSTRAIN GPD H 


\section{Projection for GPD H (HERA II)}
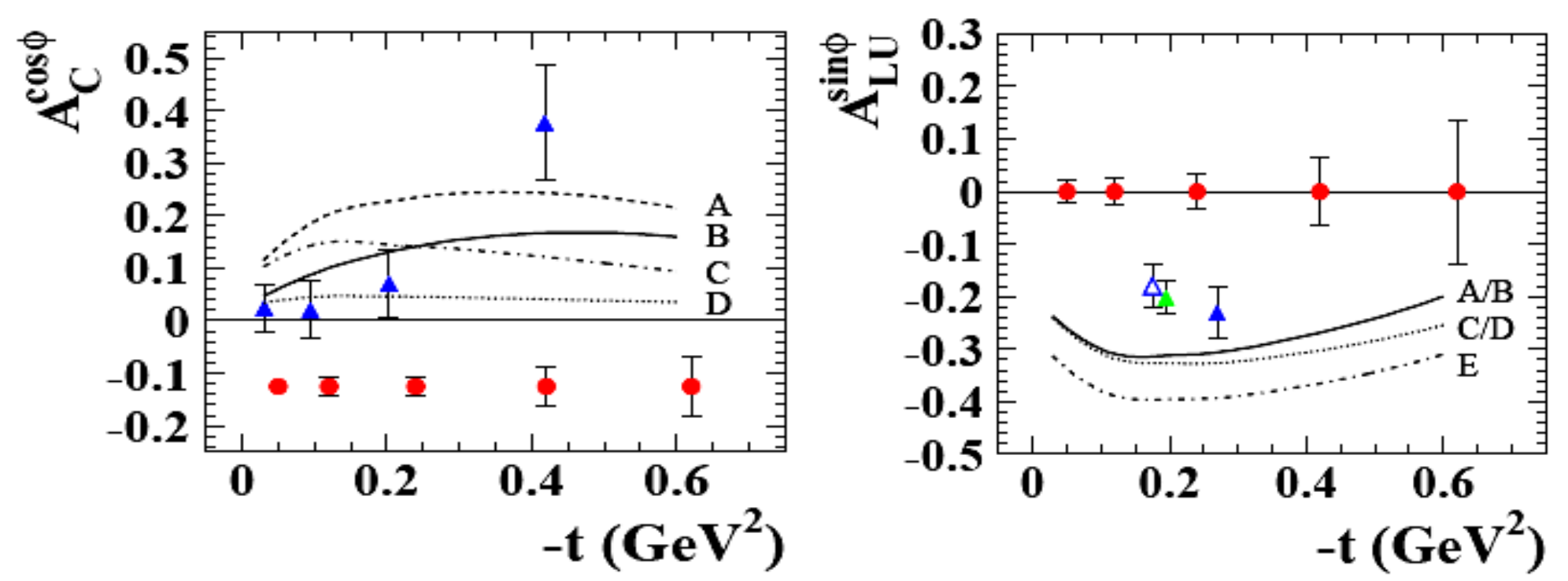

LEFT PANEL: $\Delta$ (HEP-EX/0605108) RIGHT PANEL: $\Delta$ HERMES (PRL 2001) $\triangle$ HERMES (PRELIMIMNARY HEP-EX/0212019) ACLAS (PRL 2001)

- BCA: $1 \mathrm{fb}^{-1} e^{+} \mathrm{AND} 0.25 \mathrm{fb}^{-1} e^{-}$

- BSA: $1 \mathrm{fb}^{-1} e^{+}$, BEAM POL. $\approx 35 \%$

Models: FACT. - (C/D)/REgGe - (A/B) WITh AND Without D-TERM; $b_{\text {val }}=1, b_{\text {sea }}=\infty$

BCA: HIGH SENSITIVITY TO $t$-DEPENDENCE

BSA: higheST SENSITIVITY TO $b_{\text {sea }}$ PARAMETER IN PROFILE FUNCTION(E; $\left.b_{\text {val }}=b_{\text {sea }}=1\right)$

Possibility to CONSTRain GPD $H_{u}$ 


\section{Longitudinal Target-Spin Asymmetry}

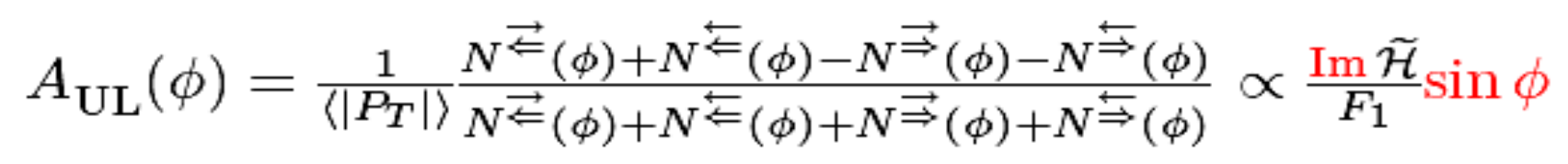
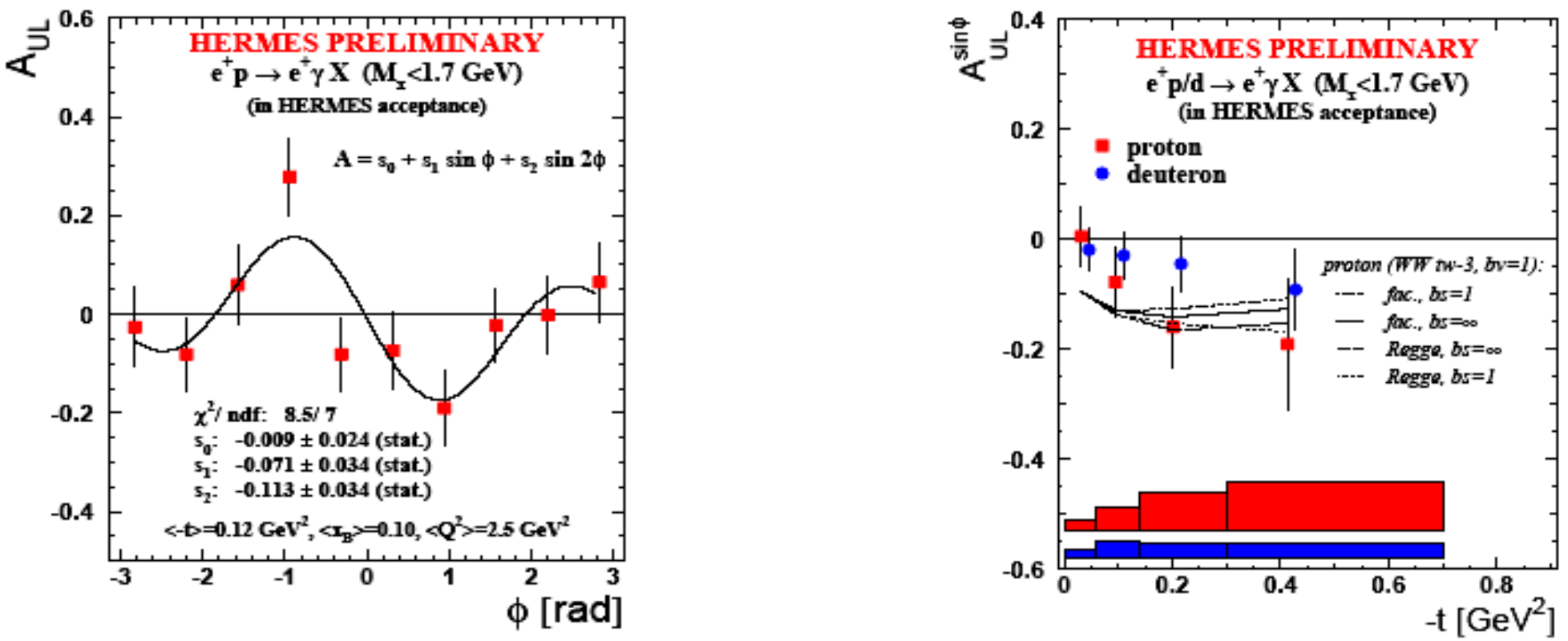

$A_{\mathrm{UL}}$ IN EXCLUSIVE BIN: ExpeCted $\sin (\phi)$ DEPENDENCE $\Longrightarrow \operatorname{Im} \widetilde{\mathcal{H}}$

-GPD MODEL: TWIST-3 IS A SIMPLE W.W. TWIST-3

- $A_{U L}^{\sin 2 \phi} \Longrightarrow(q G q)$ TWIST-3 IS MISSING ? 
$d \sigma\left(e^{+} p^{\uparrow)}-d \sigma\left(e^{+} p^{\downarrow}\right) \propto \operatorname{Im}\left[F_{2} \mathcal{H}-F_{1} \mathcal{E}\right] \times \sin \left(\phi-\phi_{\mathcal{S}}\right) \cos (\phi)+\cdots\right.$

$J_{q}=\lim _{t \rightarrow 0} \frac{1}{2} \int_{-1}^{1} d x x\left[H_{q}(x, \xi, t)+E_{q}(x, \xi, t)\right]$

$A_{\mathrm{UT}}\left(\phi,\left(\phi-\phi_{S}\right)\right)=\frac{1}{\left\langle\left|P_{T}\right|\right\rangle} \frac{N^{\uparrow}\left(\phi,\left(\phi-\phi_{S}\right)\right)-N^{\downarrow}\left(\phi,\left(\phi-\phi_{S}\right)\right)}{N^{\uparrow}\left(\phi,\left(\phi-\phi_{S}\right)\right)+N^{\downarrow}\left(\phi,\left(\phi-\phi_{S}\right)\right)}$
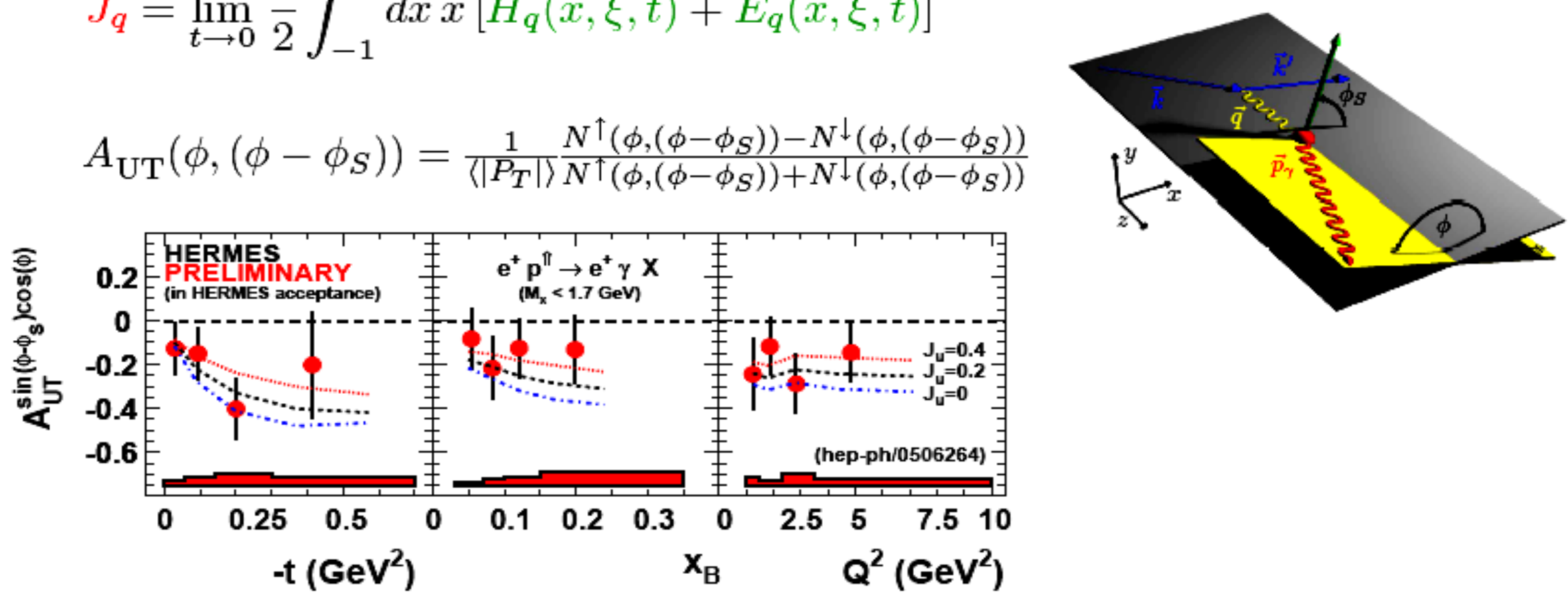

-RESUlTS FROM HERMES 2002-2004 DATA ONLY; $\simeq 60 p b^{-1}$

-Model FROM Goeke et al.: THE GPD $E$ IS UNKNOWN IN THE FORWARD LIMIT; PARAMETRIZED ACCORDING TO $\chi Q S M$ MODEL

- $A_{\mathrm{UT}}^{\sin \left(\phi-\phi_{S}\right) \cos (\phi)}$ SENSITIVE TO $J_{u}\left(J_{d}=0\right)$ 


\section{Model-Dependent Constraint on $J_{u}$ vs $J_{d}$}

-COMPARISON OF THE THEORETICAL PREDICTIONS WITH THE EXPERIMENTAL RESULTS:

$$
\chi^{2}\left(J_{u}, J_{d}\right)=\frac{\left[\left.A_{\mathrm{UT}}^{\sin \left(\phi-\phi_{S}\right) \cos (\phi)}\right|_{\exp }-\left.A_{\mathrm{UT}}^{\sin \left(\phi-\phi_{S}\right) \cos (\phi)}\right|_{V G G}\left(J_{u}, J_{d}\right)\right]^{2}}{\delta A_{\text {stat }}^{2}+\delta A_{\text {sys }}^{2}}
$$

CALCULATED IN STEP OF 0.2 IN $J_{u}$ AND $J_{d}$, INTERPOLATED BY A 5 Th ORDER POLINOMIAL.

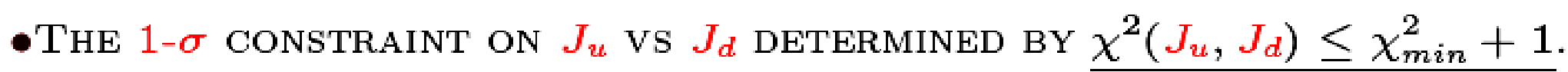

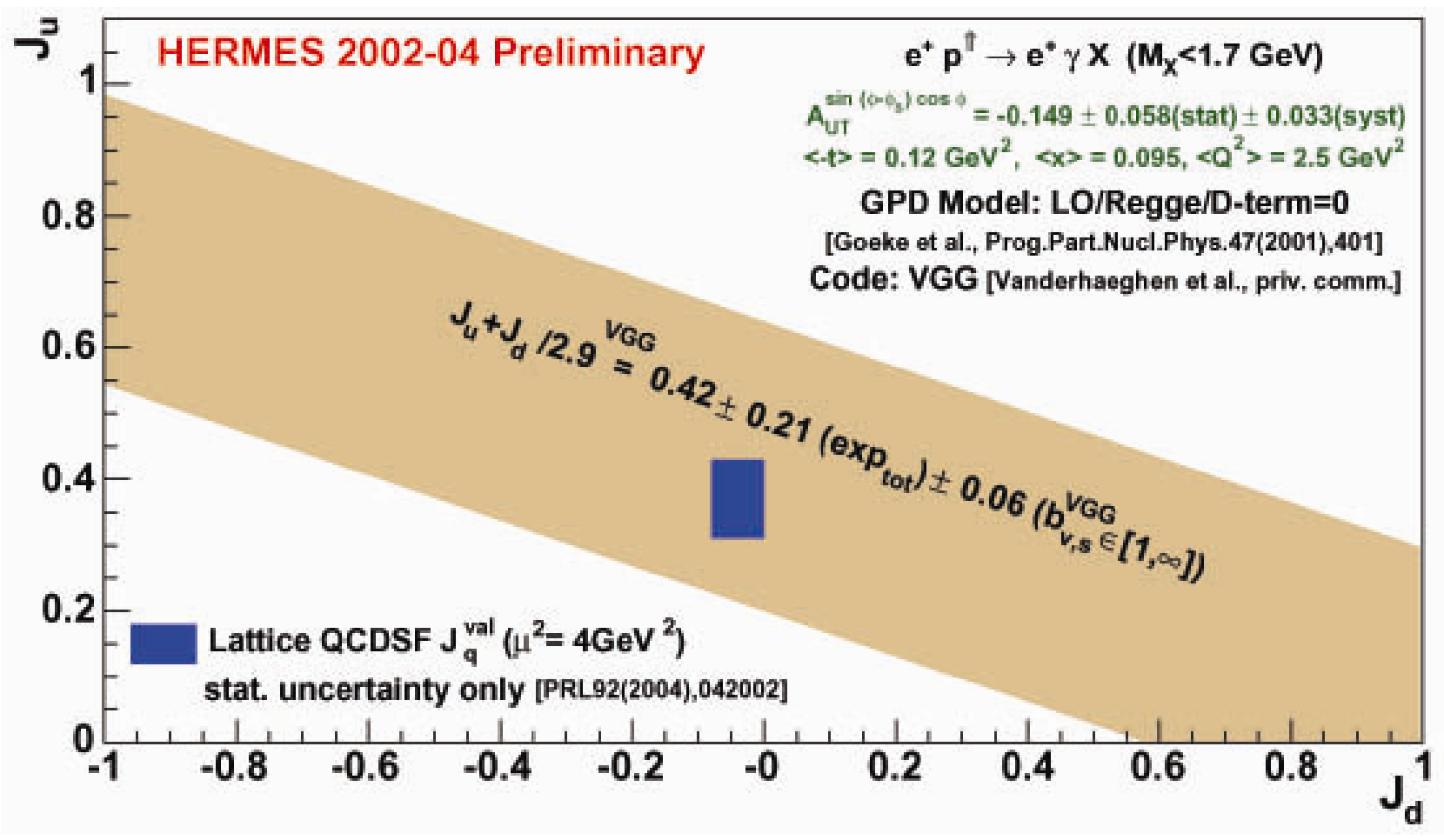




\section{Beam-Spin Asymmetry on Deuteron}

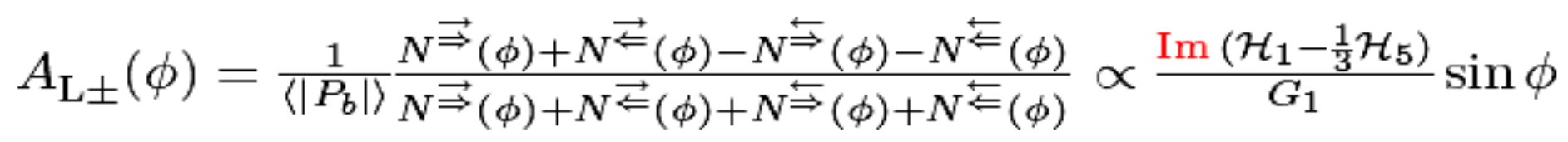
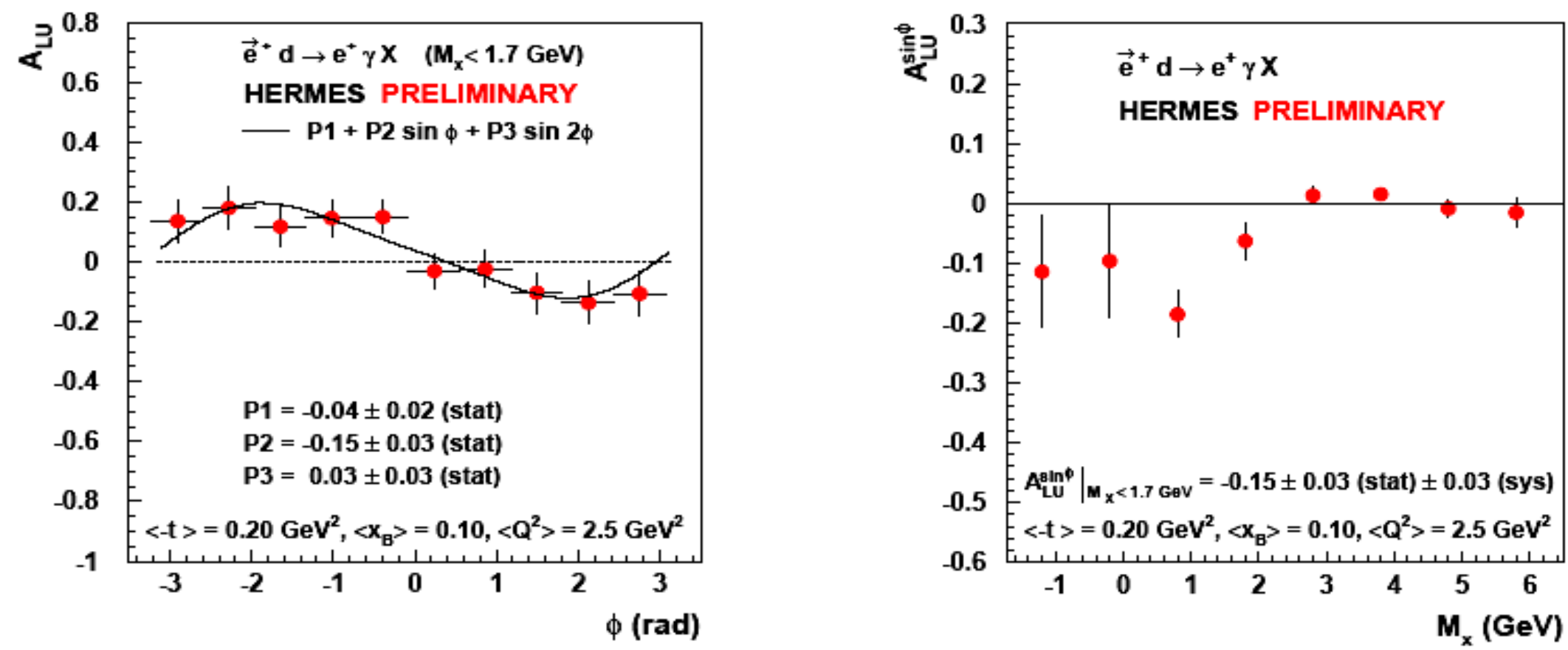

$$
\begin{aligned}
& A_{\mathrm{L} \pm, \text { Deuteron }}^{\sin \phi}=-0.15 \pm 0.03 \\
& A_{\mathrm{LU}, \text { Proton }}^{\sin \phi}=-0.18 \pm 0.03
\end{aligned}
$$

$\sin (\phi)$-MOMENTS ZERO AT HIGHER MISSING MASS 


\section{Beam-Charge Asymmetry on Deuteron}
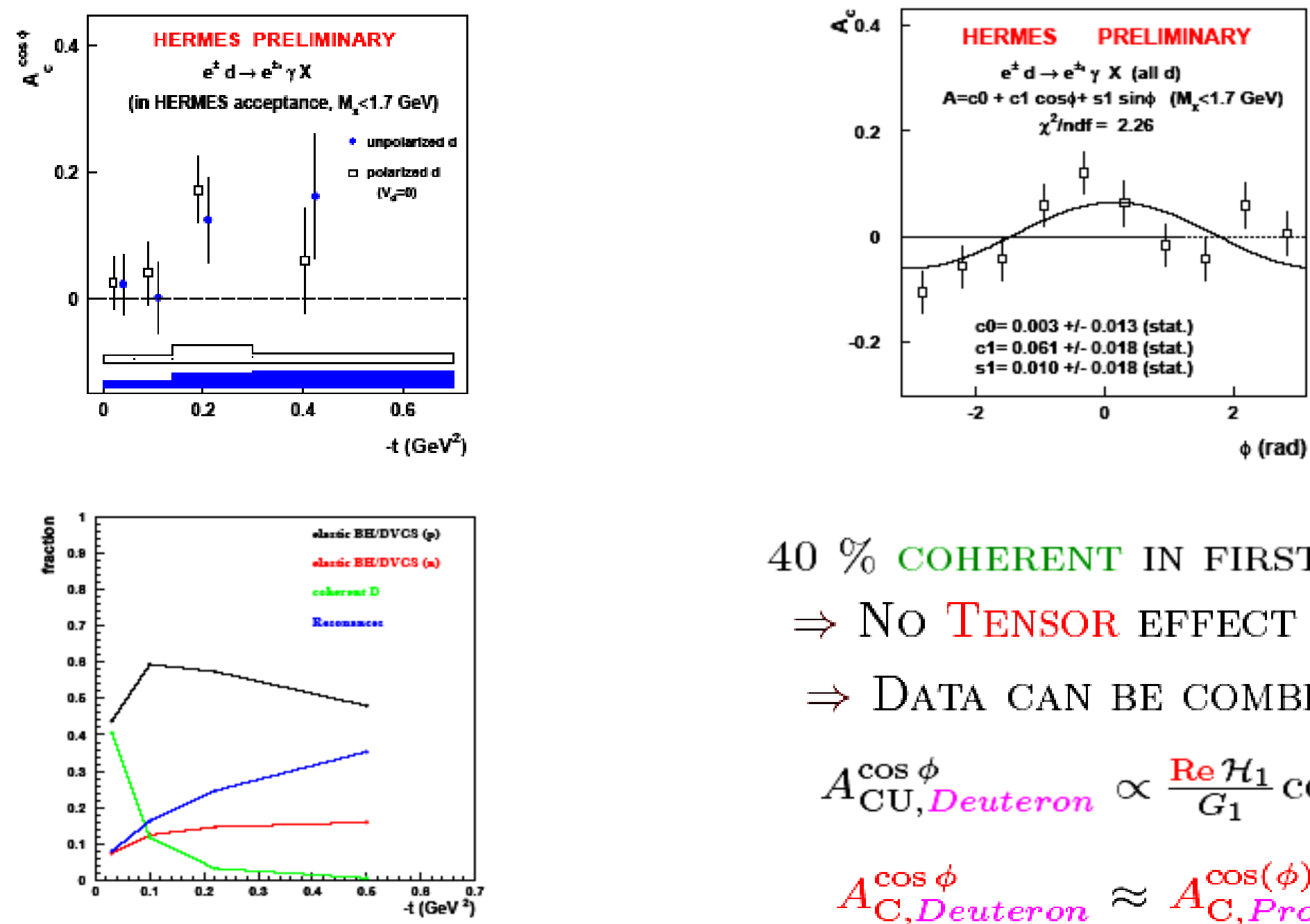

$$
\begin{gathered}
40 \% \text { COHERENT IN FIRST } t \text {-BIN } \\
\Rightarrow \text { NO TENSOR EFFECT SEEN } \\
\Rightarrow \text { DATA CAN BE COMBINED } \\
A_{\mathrm{CU}, \text { Deuteron }}^{\cos \phi} \propto \frac{\operatorname{Re} \mathcal{H}_{1}}{G_{1}} \cos \phi \\
A_{\mathrm{C}, \text { Deuteron }}^{\cos \phi} \approx A_{\mathrm{C}, \text { Proton }}^{\cos (\phi)}
\end{gathered}
$$




\section{Comparison to model calculations}

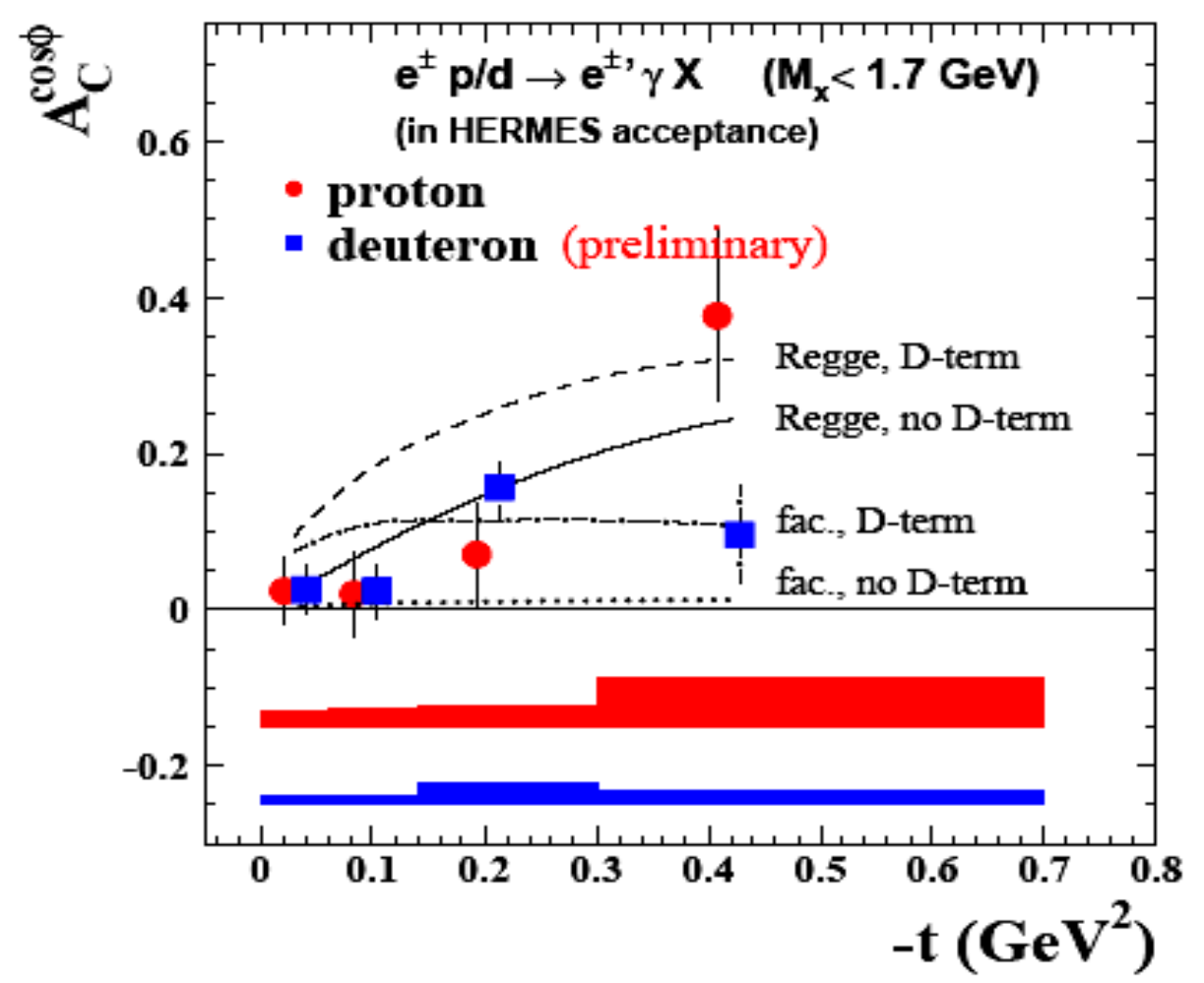

$e d \rightarrow e \gamma X$

Contributed PRocesses:

$e d \rightarrow e d \gamma$, COHERENT PROCESS

$e d \rightarrow e p n \gamma$, INCOHERENT PROCESS

$e N \rightarrow e N^{*} \gamma$, RESONANT STATES

$-t<0.7 \mathrm{GeV}^{2} \Rightarrow$ COHERENT $\approx 20 \%$

$\Rightarrow M_{X}$ Calculated via PRoton Mass

SMaller $-t \Rightarrow$ Coherent Enhanced

GPD MODELS: M. VANDERHAEGHEN et al.,

Phys. Rev. D60 (1999) 094017; K. Goeke et al.

Prog. Part. Nucl. Phys. 47 (2001) 401.

- ed COHEREnT (FIRST $t$-BIN $\approx 40 \%) \Rightarrow$ No DiffEREnCE ep AND ed

- DiffEREnCE IN LAST BIN $\Rightarrow$ NEUTRON RESONANCES, NEUTRON

SMALL $e^{-} p$ SAMPLE $\left(L \approx 10 \mathrm{~PB}^{-1}\right)$

$\Rightarrow t$-DEPENDENCE OF BCA $\rightarrow$ POSSIBLE TOOL TO CONSTRAIN GPDS 
Beam-Spin Asymmetries on Neon and Krypton
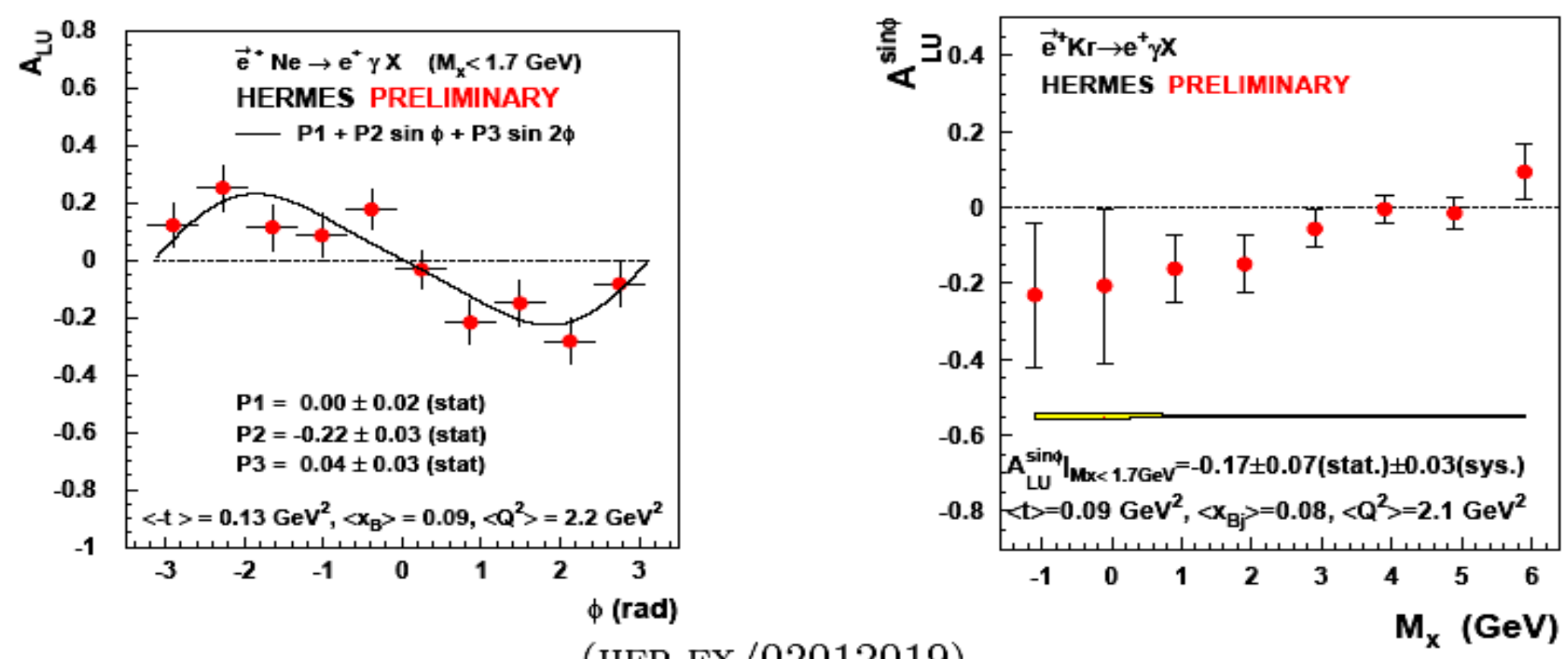

(HEP-EX/02012019)

Calculations for DVCS on (heavier) Nuclei (Kirchner, Müller, hepPH/0302007, GUZEY, STRIKMAN, HEP-PH/0301216, ...)

- Sizeable BSA; Comparison TO THEORY $\Longrightarrow$ SEPARATE COHERENT AND INCOHERENT PARTS $\Longrightarrow$ THROUGH $t$-DEPENDENCE OF BSA (IN PROGRESS) $\Longrightarrow$ A-DEPEndenCE OF BSA (H, D, NE, KR, XE) And BCA (H, D, KR, $\mathrm{XE}) \Longrightarrow$ GENERALIZED EMC-EFFECT IN NUCLEAR DVCS 
Summary and Outlook

Azimuthal Asymmetries $\Rightarrow$ DVCS-Amplitudes $\Rightarrow$ GPDs

$\Rightarrow$ Structure of Nucleons $\left(L_{q}\right)$

EXISTING HERA I DATA ON $H$ :

- BEAM-SPIN AsYMMETRY $\Rightarrow \operatorname{Im} H$

- Beam-Charge Asymmetry $\Rightarrow \mathrm{Re} H$

- First constraints on GPD MODEls

LONGITUDINALLY POLARIZED $H \Rightarrow$ LTSA $\Rightarrow \operatorname{Im} \widetilde{H}$

TRANSVERSE POLARIZED $H \Rightarrow$ TTSA $\Rightarrow E$

- First model-DePEndent Constraint on $J_{u}$ Vs $J_{d}$

\section{OUTLOOK}

- InCluding THE 2005 DATA WILL DOUBLE THE STATISTICS FOR TTSA

- HERA II(2002-2007) DATA ON $H$ PROBABLY MAP OUT THE GPD $H_{u}$

- IMPROVEMENT OF $t$-RESOLUTION WITH Recoll Detector

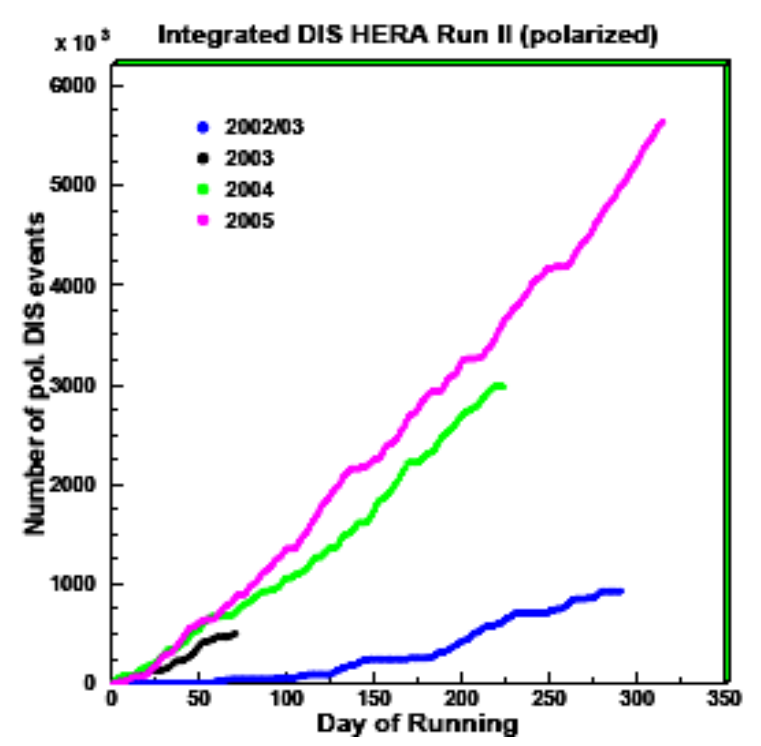




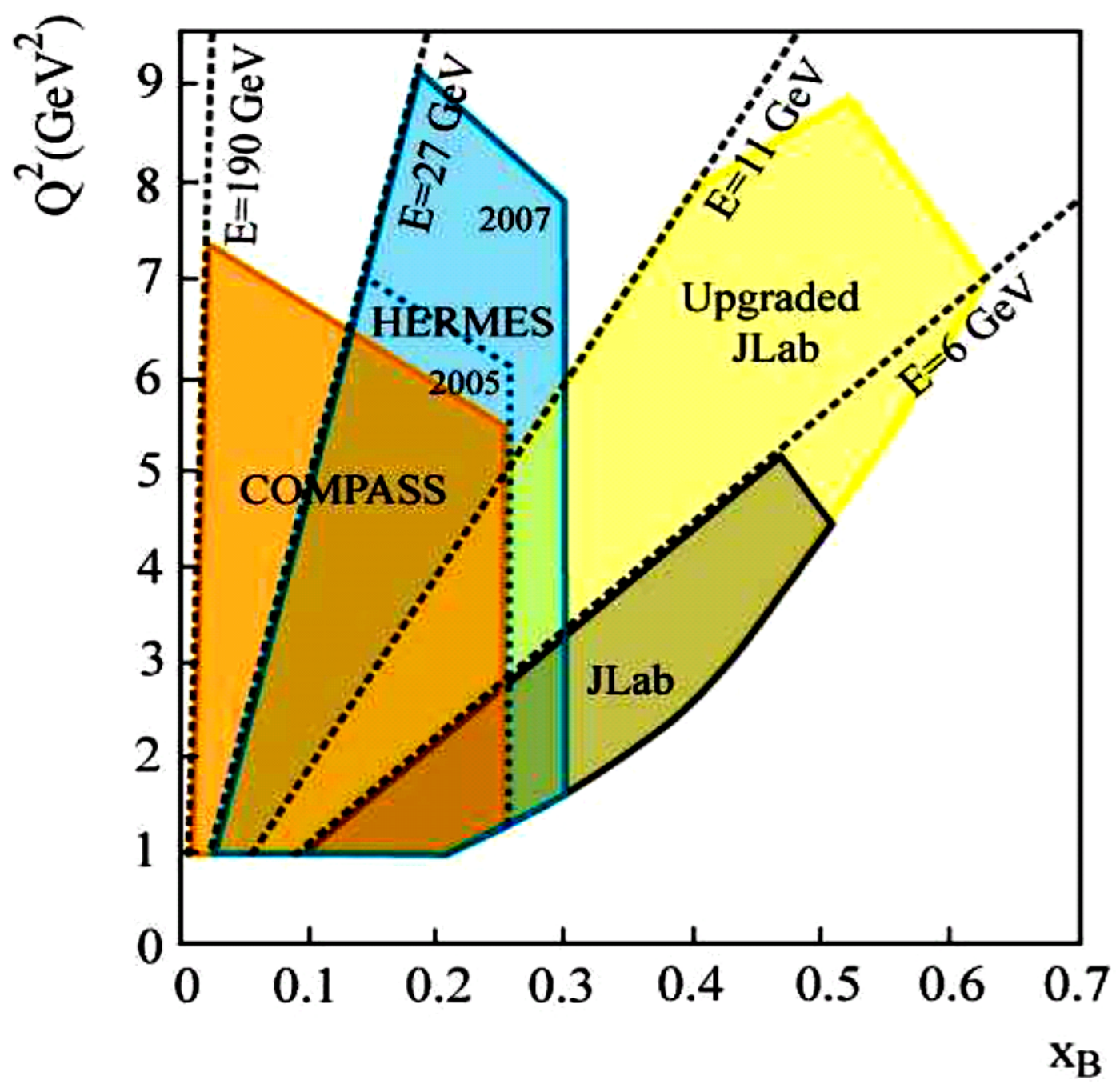


- Instead of having an initial electromagnetic process, a final state electromagnetic process is selected $=>$ Generalizing the GPDs for $\mathrm{P} \overline{\mathrm{P}}$ interaction

-At intermediate energies (S $10 \mathrm{Gev}$ ) Handbag diagram

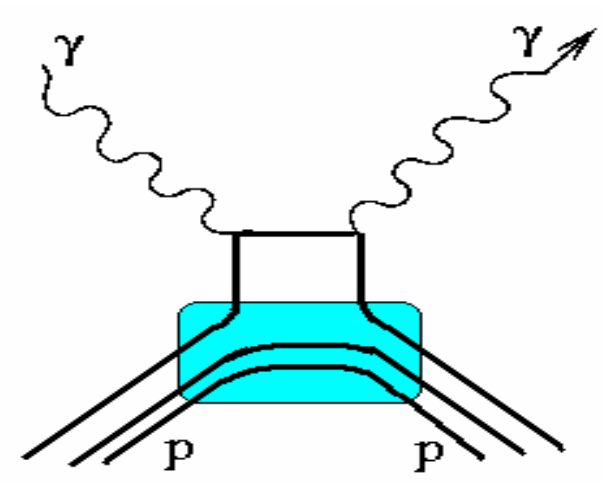

Compton scattering

- PANDA-PAX programs at GSI-FAIR. Handbag approach can be probed over larger range of energy and perhaps with higher precision. Polarization of $\mathrm{P}$ and $\overline{\mathrm{P}}$ is helpful.

- Detectors should be ready to measure different kind of exclusive reaction at FAIR.

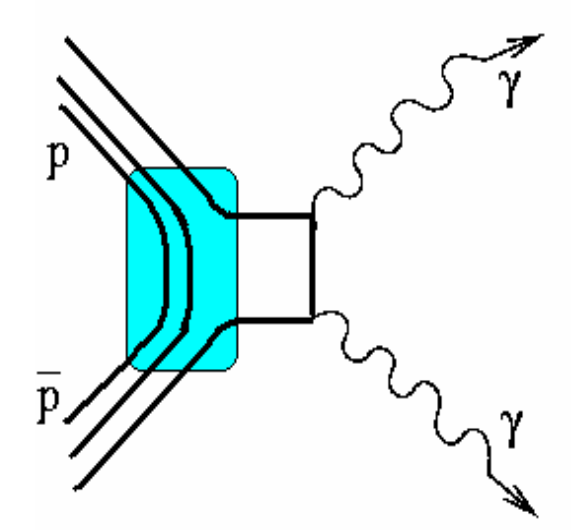

Annihilation 